\title{
Finite Element Modelling and Investigation of the Process Parameters in Deep Rolling of AISI 4140 Steel
}

\author{
Nataliya Lyubenova* and Dirk Baehre \\ Saarland University, Institute of Production Engineering, Saarbruecken 66123, Germany
}

\begin{abstract}
The application of beneficial residual stresses in components is often used to increase their fatigue life and resistance to FOD (Foreign object damage). There are several production processes having the main aim to induce beneficial compressive residual stresses at the surface and on the sub-surface level in components. As examples can be mentioned the shot peening, autofrettage, laser shock peening, ultrasonic impact treatment and DR (Deep rolling) processes. The prediction and measurement of the induced residual stresses is always difficult and a time-consuming and therefore, the employment of the FEA (Finite element analysis) to model such processes can be very profitable. In this article the explicit module of the FE-Code ABAQUS 6.13 was applied to model the DR process on a plane geometry. The input parameters applied force, number of overturns and percentage of overlapping are varied and their influence on the resulted depth profile of residual stresses is commented on.
\end{abstract}

Key words: Residual stresses, mechanical surface treatment, deep rolling, finite element simulation.

\section{Introduction}

In the modern industry the targeted application of compressive (= negative) residual stresses in highly stressed components is gaining great importance. Compressive residual stresses are often used as design instrument in order to increase the fatigue life of the components and to improve their resistance to foreign object damage [1]. It is well known that parts, being exposed to tensile (= positive) static as well as dynamic loads are prone to crack formations. When compressive residual stresses are superimposed to the operating tensile loads the former reduce the total loading, thus retarding the surface cracking and preventing the initiation of new cracks.

There are numerous production processes which are able to introduce pronounced compressive residual stresses differing in the depth profile. Typical examples are shot peening, autofrettage, laser shock peening, ultrasonic impact treatment and deep rolling.

Well-recognized is the DR (Deep rolling) process that has initially attracted the interest of the scientific

"Corresponding author: Nataliya Lyubenova, M. Sc., research fields: residual stresses and manufacturing processes. community in the thirties of the last century [2]. Investigations of the process in the seventies and eighties led to the clear consideration that it is able to improve the fatigue life of the treated components. According to the Association of German Engineers VDI guideline 3177 [3], DR is classified as fine surface rolling method, next to finishing and size rolling. In the available literature different terms, like deep rolling, deep cold rolling, roller burnishing, low plasticity burnishing and others, are used to describe processes being similar in mechanism and results. The main advantage of DR is the high amount and depth of the achieved compressive residual stresses which in some materials can exceed $1 \mathrm{~mm}$ [4]. Another advantage of the process is the reduction of the surface roughness which prevents the crack formation and -propagation. In steel the roughness after treatment with DR can be well below $1 \mu \mathrm{m}$ (Rz). A deep layer of strain hardening and plastic deformation also belongs to the properties of the treated component, thus contributing to its longer life time.

Although well-known, DR is still investigated due to the large amount of input parameters which lead to great differences in the results. Schulze [5] offers a 
comprehensive classification of the DR parameters by dividing them into work piece-, tool-, process- and device parameters, see Fig. 1. Some of them, like the applied force or pressure, the number of overturns, the percentage of overlapping, the properties and geometry of the work piece, etc., directly influence the quality of the surface as well as the depth and amount of the achieved residual stresses.

Wong [6] varied the DR pressure and found out that the increasing of the applied pressure leads to higher and deeper penetrating compressive residual stresses.

FE (Finite element) Modelling is a powerful instrument to model complex processes, e.g. DR, and offers a good opportunity to vary and investigate numerous input- and output parameters. Manoucherhrifar et al. [7] used the explicit dynamic algorithm in ABAQUS 6.10 to model different DR input parameters. They found out that for some fixed boundary conditions the resulted from the FE-simulation residual stresses are in good agreement with those experimentally derived. Still, the variation of other boundary conditions, as for example the friction coefficient, led to uncertain residual stress depth profiles. The FEM (Finite element method) coupled with the BEM (Boundary element method) was employed by Bärker et al. [8] in order to simulate the residual stresses induced by DR in turbine blades. The FEM/BEM coupling was used to compute a large scale model offering low computing time and high result accuracy. They compared the modelled residual stresses with experimental data and stated that the former are in quantitatively good agreement with the experimentally derived residual stresses.

Nevertheless, when modelling the DR process the simplification of the boundary conditions, material behaviour or contact formulation can cause misleading results. Therefore, in this paper the Explicit module of the FE-Code ABAQUS 6.13 is applied to model the DR process on a plane geometry. The input parameters applied force, number of overturns and percentage of overlapping are varied and their influence on the induced residual stresses is commented on.

\section{Deep Rolling Principle and Application}

The main principle of DR can be described as follows: the DR tool, which can be a hydrostaticallyor spring-supported roller or sphere [9],

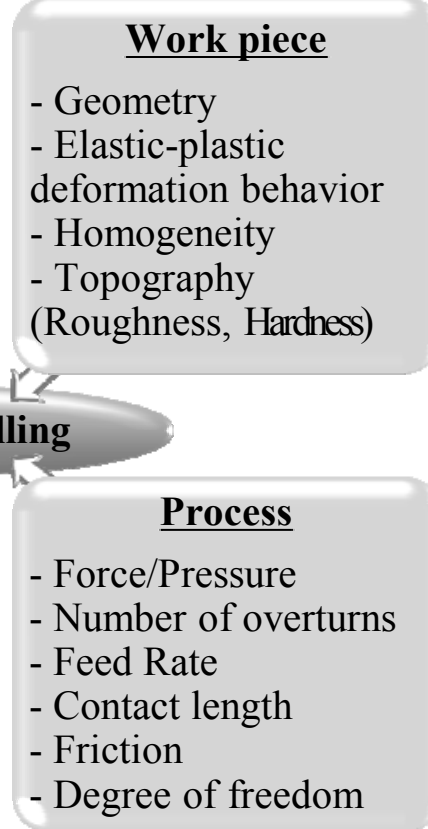

Fig. 1 Classification of the DR input parameters [5]. 
is pressed with a defined force or -pressure against the work piece. This leads to a maximum equivalent stress formed beneath the work piece's surface. If this stress is below the yield point of the material, the stress field along the contact normal is governed by the elastic stresses theories of Hertz and Föppl. If the applied force or pressure is further increased, the equivalent stress reaches the yield point of the material, leading to plastic deformations beneath the DR roller or sphere [10]. At the opposite side of the treated surface only elastic deformations exist. After the force or pressure application different treatment strategies, similar to the milling strategies, as varying the rolling path, can be used. All of the strategies have in common that the DR tool is always freely rotating. At the end of the treatment the applied force or pressure is released and the elastically deformed part of the work piece strives to return to its original state but is prevented from the plastically deformed area. This inhomogeneous deformation leads to a generation of compressive residual stresses at the treated surface, being compensated with tensile stresses at other parts of the work piece [11]. The basic DR process setup for plane geometry can be seen in Fig. 2.

In Fig. 3, some typical applications of the DR process can be observed. Fig. 3a shows DR of a wheel flange where the sharp edge is rounded and compressive residual stresses are induced, thus reducing the stress concentration in this area. By means of DR the tensile residual stresses induced by welding, see Fig. 3b, can be neutralized or even shifted to the compression level. The lifetime of valves is also increased when using the DR process (Fig. 3c). Also often, the tension screws that support the aircraft's engine are treated with DR to increase their lifetime, see Fig. 3d. In mass production the DR process is applied to turbine blades to increase their resistance to a foreign object damage [12].

\section{Finite Element Modelling of DR Process}

The investigations presented in this article are performed using the FE Code ABAQUS 6.13. The Explicit module of the program was employed in order to model the dynamics of the DR process. This module offers the possibility to perform a nonlinear dynamic stress/displacement analysis as well as numerous contact modelling techniques.

\subsection{Geometries}

The presented investigations use a full 3D (3 dimensional) model of the DR processes. Due to the anisotropy of the residual stresses induced in rolling

DR tool

with applied pressure

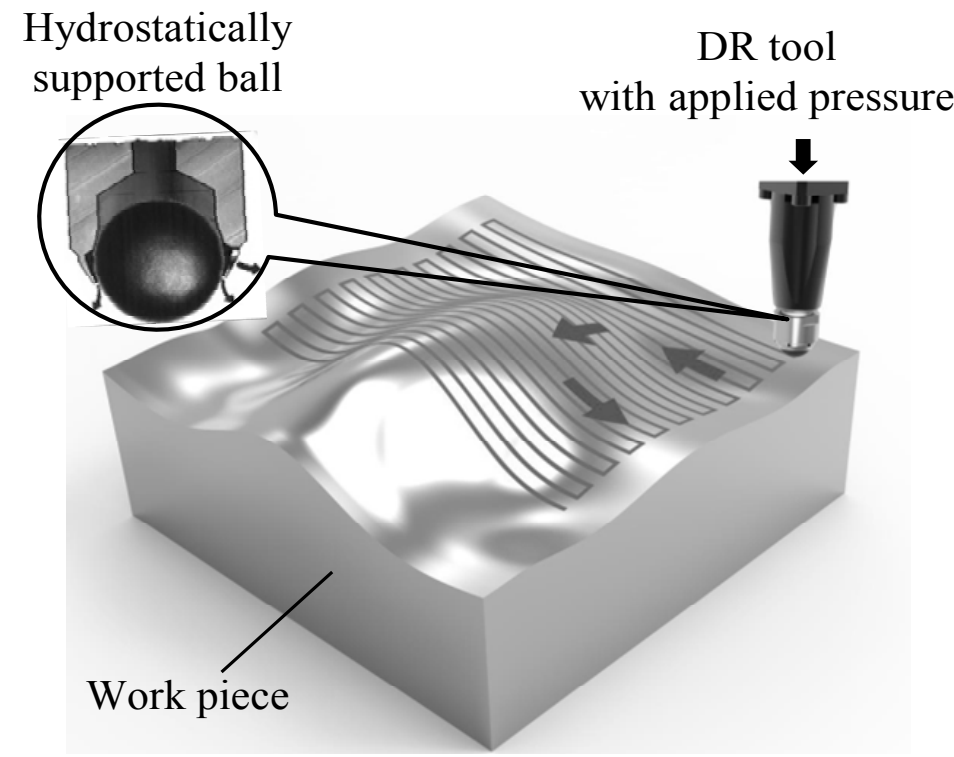

Fig. 2 DR basic setup. 

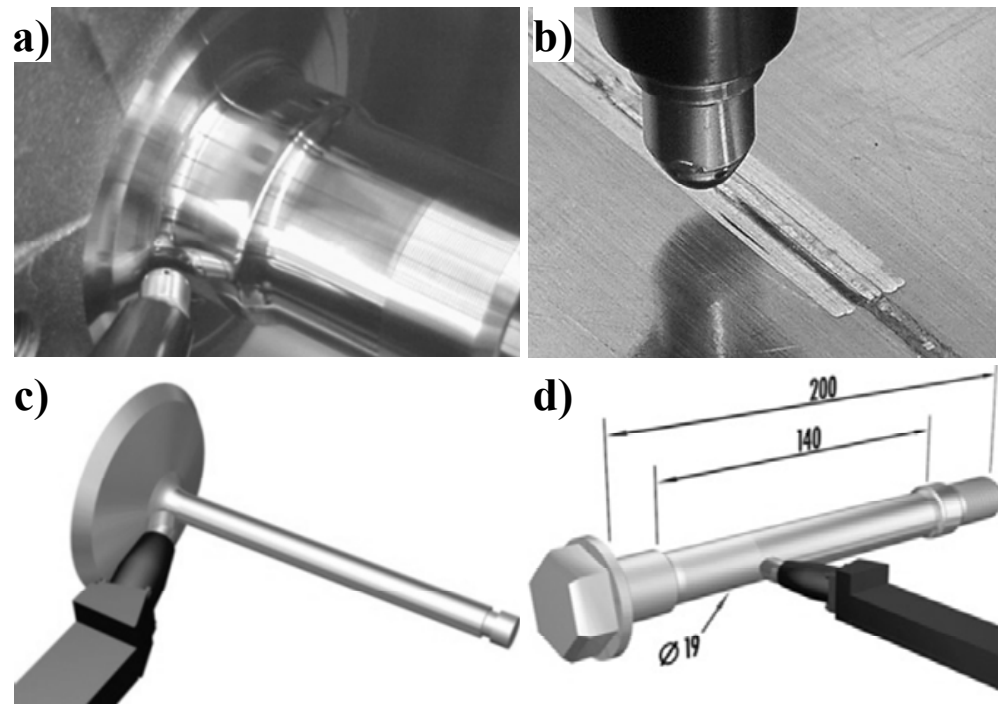

Fig. 3 Deep rolling application examples: (a) DR of a wheel flange, (b) DR of a welded joint, (c) DR of a valve and (d) DR of a tension screw [13-14].

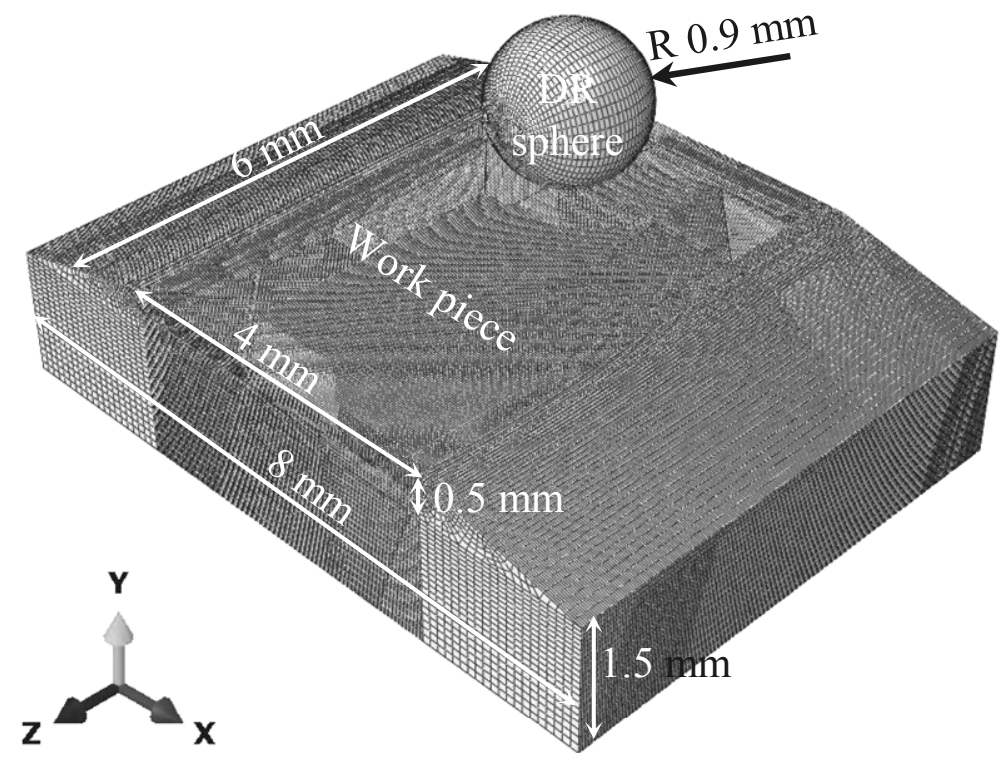

Fig. 4 DR geometries.

Table 1 Work piece material data.

\begin{tabular}{ll}
\hline $\begin{array}{l}\text { Work piece material data - DIN EN } 10002 \text { uniaxial tensile } \\
\text { test data for AISI 4140 }\end{array}$ \\
\hline Young's modulus & $211,000 \mathrm{MPa}$ \\
Yield strength & $973 \mathrm{MPa}$ \\
Ultimate strength & $1,073 \mathrm{MPa}$ \\
Fracture strain & $4.72 \%$ \\
Poisson's ratio & 0.28 \\
\hline
\end{tabular}

and transverse direction, 2 dimensional simulation will not show the full stress distribution. In Fig. 4, the dimensions of the employed 3D work piece and DR sphere are shown. For all of the presented investigations the work piece and the sphere dimensions were fixed.

\subsection{Material Definition}

The work piece material was defined as a high strength steel AISI 4140. Its physical data as well as its chemical composition are shown in Tables 1 and 2 respectively. The DR sphere was modelled as rigid body with defined mass and moment of inertia.

The material data was derived by means of an uniaxial tensile test. To implement the data into the FE 
Table 2 Chemical composition in weight \% of the steel AISI 4140.

\begin{tabular}{lllllllll}
\hline \multicolumn{2}{l}{ Chemical composition in weight \% of the steel AISI 4140} \\
\hline $\mathrm{C}$ & $\mathrm{Mn}$ & $\mathrm{Si}$ & $\mathrm{P}$ & $\mathrm{S}$ & $\mathrm{Cr}$ & $\mathrm{Mo}$ & $\mathrm{Se}$ & $\mathrm{Ca}$ \\
\hline 0.39 & 0.86 & 0.22 & 0.013 & 0.08 & 1.07 & 0.20 & 0.023 & 0.002 \\
\hline
\end{tabular}

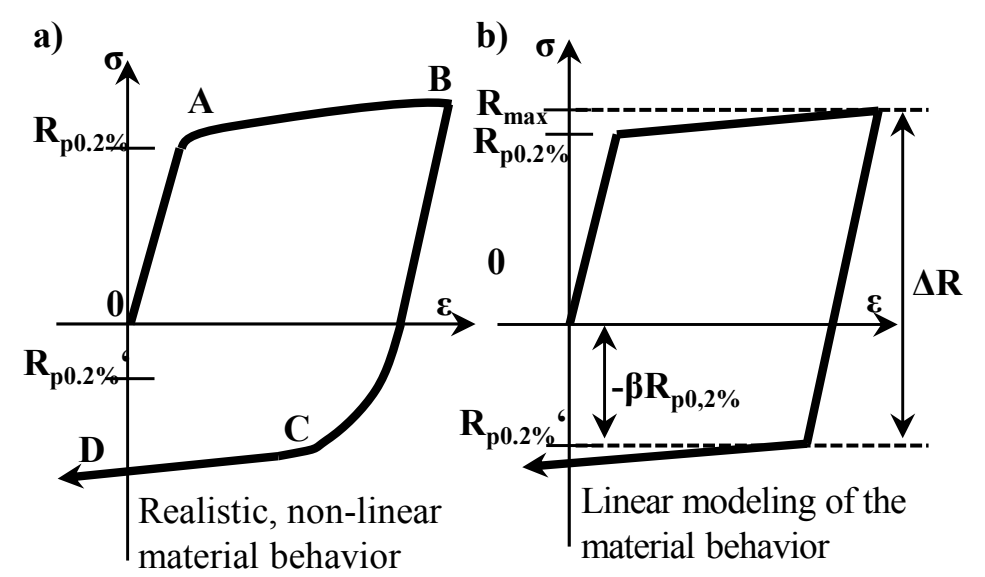
0-A: Elastic load
A-B: Plastic load + hardening
B-C: Elastic unloading
C-D: Unloading with Bauschinger-Effect and hardening

1. $\Delta \mathbf{R}=\mathbf{R}_{\max }+\boldsymbol{\beta} \mathbf{R}_{\mathrm{p} 0.2 \%}$ - General formulation for empirical consideration of the Bauschinger effect

$\mathbf{2 .} \Delta \mathbf{R}=\mathbf{2 R}_{\max }$ - Linear elastic, plastic with isotropic hardening

3. $\Delta R=2 R_{p 0.2 \%}$ - Linear elastic, plastic with kinematic hardening

Fig. 5 Work piece material behaviour definition: (a) realistic, non-linear material behaviour and (b) linear modelling of the material behavior [19].

model the material model of Huang was employed [15]. He divides the stress-strain curve into for regions, see Fig. 5a. The region 0-A describes the initial tensile loading regime during which the steel behaves elastically up to the yield point $\mathrm{R}_{\mathrm{p} 0.2 \%}$. Here, the material behavior follows Hooke's law. Then, the material behaves plastically, region A-B. This region can involve significant non-linearity. It is followed by the unloading elastic regime $\mathrm{B}-\mathrm{C}$ during which the steel behaves elastically up to the yield point $R_{p 0.2 \%}$. The last phase of the stress-strain curve is the C-D region where the material behaves plastically. This phase exhibits also a significant non-linearity. For a simplification of the material modelling a linear representation can be employed. In Fig. 5b, different linear approximations are shown. The first simplification considers the Bauschinger Effect [16] and shows the closest correspondence to the real material behavior. The disadvantage is that the Bauschinger coefficient $\beta$ needs to be empirically derived. The second option - linear elastic, plastic with isotropic hardening - does not consider the Bauschinger effect and this can lead to an overestimation of the modelled residual stresses. Demurger et al. [17] confirms also that compared to their kinematic hardening model, the model using an 
isotropic hardening overestimated the FE-modelled residual stresses. The third possibility to model the material behavior is to employ a bi-linear kinematic hardening model. It is able to represent the Bauschinger effect and therefore, it was used in the presented investigations. This model was successfully implemented in the FE simulations of a similar mechanical surface treatment, namely Autofrettage process [18, 19]. Still, it has to be considered that the real material behavior includes non-linear regions and their linear simplifications can be a source of uncertainties.

\subsection{Contact Formulation}

The DR process includes always a contact between the work piece and the tool (here: DR sphere). The proper description of the contact is necessary to avoid an artificial deformation of the treated surface. Here, the "contact pair" option was used, as the DR sphere surface was defined as master and the work piece's surface as slave. The friction coefficient was fixed to 0.1 and an isotropic frictional directionality was chosen. Additionally, a penalty option was added. This allows defining an elastic slip between both surfaces where the work piece is allowed to elastically deform, as the DR sphere is a rigid body. The elastic slip was fixed to $0.0005 \%$ of the element size. The application of a small elastic slip is recommended by the ABAQUS User's Manual [20], as it improves the solution accuracy.

\subsection{Meshing Strategies}

For the proper meshing of the work piece initially a convergence study was conducted. After the initial increasing of the measured von Mises maximal stress, further increasing of the mesh density led to a plateau of the stresses reached at element size of $0.04 \mathrm{~mm}$. The calculation time was about $20 \mathrm{~min}$. In order to investigate the depth distribution of residual stresses more precisely the work piece thickness was divided into three partitions, see Fig. 6. Partition 1 has a fixed element size of $0.025 \mathrm{~mm}$. For the second and third partition a progressive meshing strategy was employed, offering the opportunity for smooth decreasing of the mesh density out of the region of interest (along -Y direction). In these two regions the element size increased progressively from $0.025 \mathrm{~mm}$ to $0.05 \mathrm{~mm}$ and finally to $0.1 \mathrm{~mm}$. The work piece was meshed using hexahedral C3D8R elements. These are 3 dimensional, 8 nodes elements with reduced integration. For the meshing of the DR sphere, 2880 linear quadrilateral elements type R304 were employed.

\subsection{Modelling Steps and Boundary Conditions}

The FE simulation of the DR process was performed using 7 modelling steps, see Table 3 . In the initial step the fixation of the work piece was realized. After that a contact between the work piece and the DR sphere was established, using a relatively small normal force. The third step was the application of the

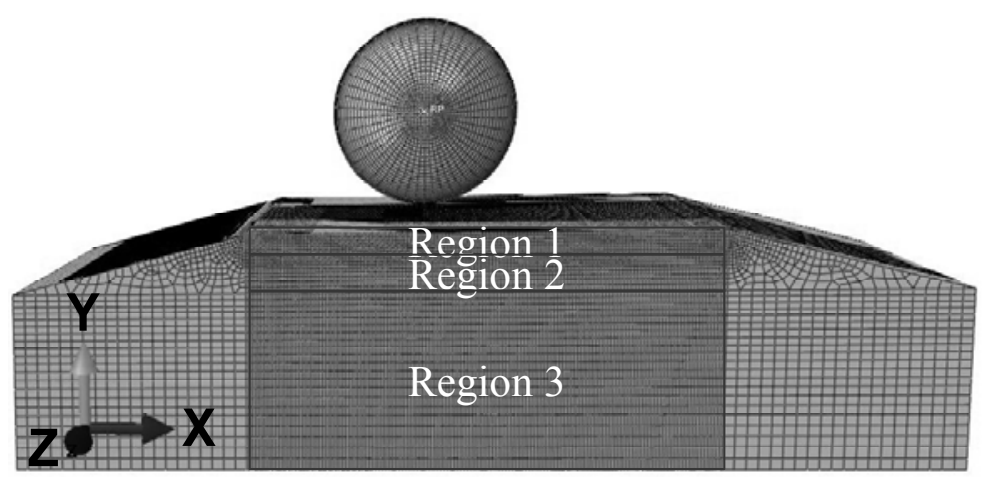

Fig. 6 Meshing strategy of the work piece. 
Table 3 Modelling steps and boundary conditions applied to the DR sphere.

\begin{tabular}{llllllll}
\hline \multirow{2}{*}{ Modelling steps } & \multicolumn{3}{c}{ Movement } & \multicolumn{3}{c}{ Rotation } & \multicolumn{2}{c}{ Applied force } \\
\cline { 2 - 7 } & $\mathrm{X}$ & $\mathrm{Y}$ & $\mathrm{Z}$ & $\mathrm{X}$ & $\mathrm{Y}$ & $\mathrm{Z}$ & \\
\hline Initial & - & + & - & - & - & - & - \\
Contact & - & + & - & - & - & - & $-\mathrm{Y}=5 \mathrm{~N}$ \\
Apply force & - & + & - & - & - & - & $-\mathrm{Y}=25$ to $100 \mathrm{~N}$ \\
Movement 1 & - & - & + & + & - & - & $-\mathrm{Y}=25$ to $100 \mathrm{~N}$ \\
Movement 2 & + & - & - & - & - & + & $-\mathrm{Y}=25$ to $100 \mathrm{~N}$ \\
Movement 3 & - & - & + & + & - & - & $-\mathrm{Y}=25$ to $100 \mathrm{~N}$ \\
Release force & - & + & - & - & - & - & Deactivated \\
\hline
\end{tabular}

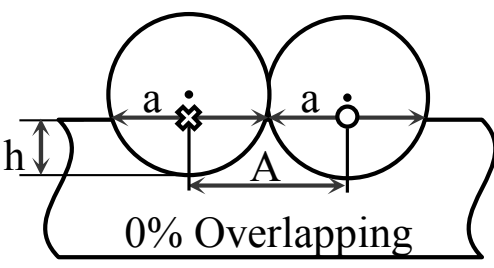

$$
\mathrm{a}=2 \sqrt{h(2 r-h)}
$$

Fig. 7 Definition of the percentage of overlapping.

DR force which varied for the different simulations. The next three steps were the DR trajectories in $\mathrm{Z}, \mathrm{X}$ and $-Z$ directions. The last step simulated the releasing of the DR force.

\subsection{Definition of the DR Input and Output Parameters}

In the presented investigations a few DR input parameters were varied. First, the applied DR force was varied from 25 to $100 \mathrm{~N}$. The levels were chosen in a way that they corresponded to a low DR pressure (a force of $25 \mathrm{~N}$ is equal to 98 bar) and a high DR pressure (a force of $100 \mathrm{~N}$ is equal to $390 \mathrm{bar}$ ).

The next varied DR input parameter was the percentage of overlapping. The definition of the parameter is shown in Fig. 7. Here, the depth penetration $h$ of the DR sphere was measured and the distance $a$ calculated. After that the overlapping parameter A was defined as a percentage of the distance $a$.

The last varied DR input parameter is the number of overturns. It describes how many times a DR was applied to the same treatment area.

The investigated DR output parameters were the longitudinal (along the rolling direction) and transverse residual stresses. The depth profile of the residual stresses was obtained as in the middle of the first rolling trajectory (Step - Movement 1) in Z direction a measurement path was defined from the surface toward -Y direction in depth.

\section{Results}

4.1 Effect of the Applied DR Force on the Depth Distribution of Induced Residual Stresses

In Fig. 8, the depth distribution of transverse residual stresses when varying the DR force is shown. Here, it is visible that the application of a higher force leads to higher magnitude as well as deeper distribution of the induced residual stresses. Typically for the transverse stresses induced by DR, their maximum lays beneath the treated surface.

The diagram in Fig. 9 depicts the comparison of the longitudinal residual stresses after variation of the DR force. Here, the same as for the transverse residual stresses behavior can be observed but the maximum of the stresses is right on the treated surface. This anisotropy of the residual stresses achieved in longitudinal and transverse direction is mentioned in 


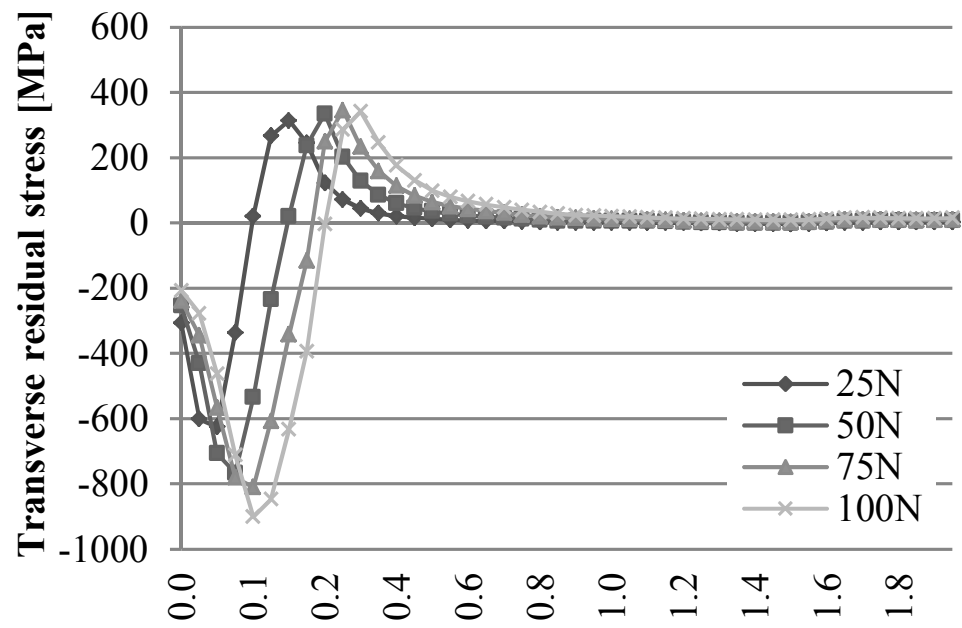

Depth [mm]

Fig. 8 Variation of the DR force - transverse residual stresses.

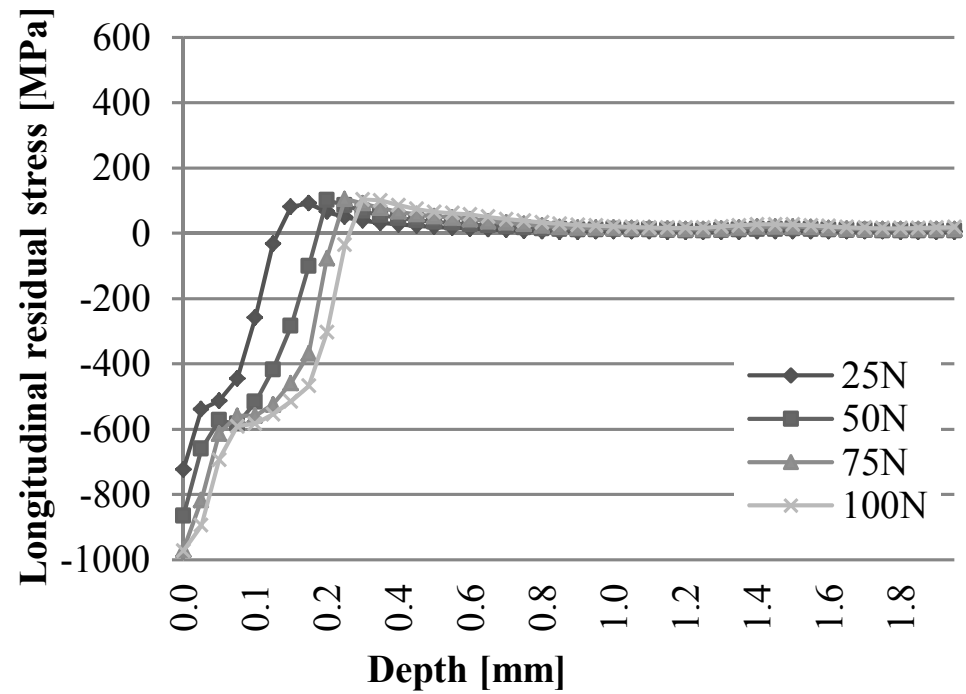

Fig. 9 Variation of the DR force - longitudinal residual stresses.

several publications $[6,7]$.

\subsection{Effect of the Variation of the Number of Overturns} on the Depth Distribution of Induced Residual Stresses

The variation of the number of overturns is plotted in Fig. 10. Here, the repetition of the DR process does not lead to an increasing of the maximal induced compressive residual stresses. It can be seen that they even slightly decrease. Still, as a positive effect it can be observed that the more repetitions are performed, the deeper the compressive residual stresses penetrate, reaching a depth of approximately $0.5 \mathrm{~mm}$ at 5 overturns. Compared to those achieved without overturn, the layer with compressive residual stresses achieved with 5 overturns is double thicker.

In Table 4, the calculated area with compressive residual stresses when varying the overturn parameter is plotted. Here, it is more visible that the increasing of the number of overturns leads to a greater area with compressive residual stresses and the area is even slightly exponentially increasing. 


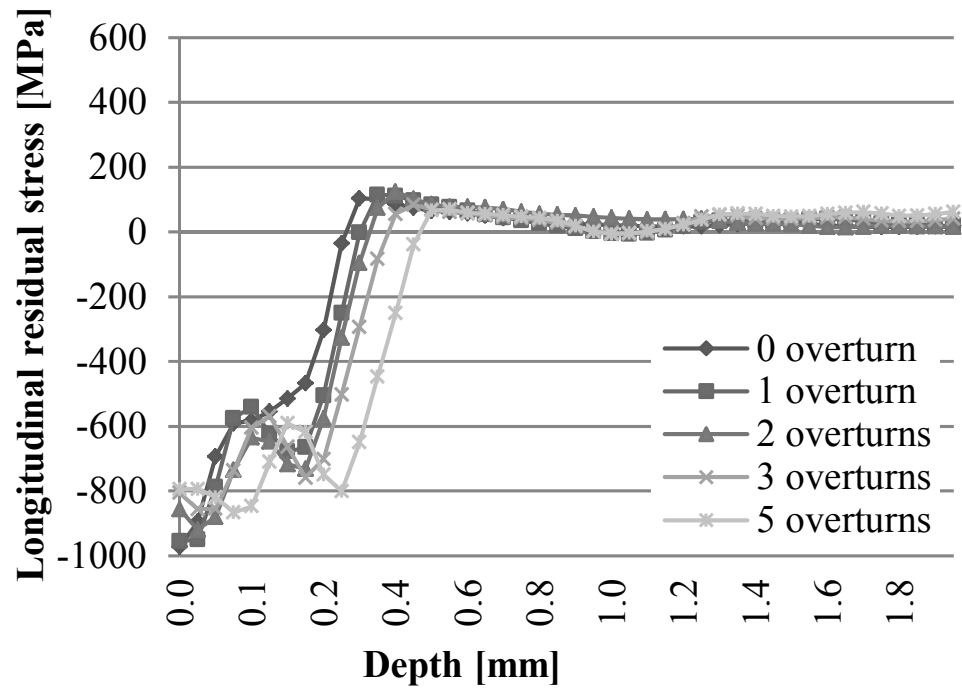

Fig. 10 Variation of the number of overturns - longitudinal residual stresses.

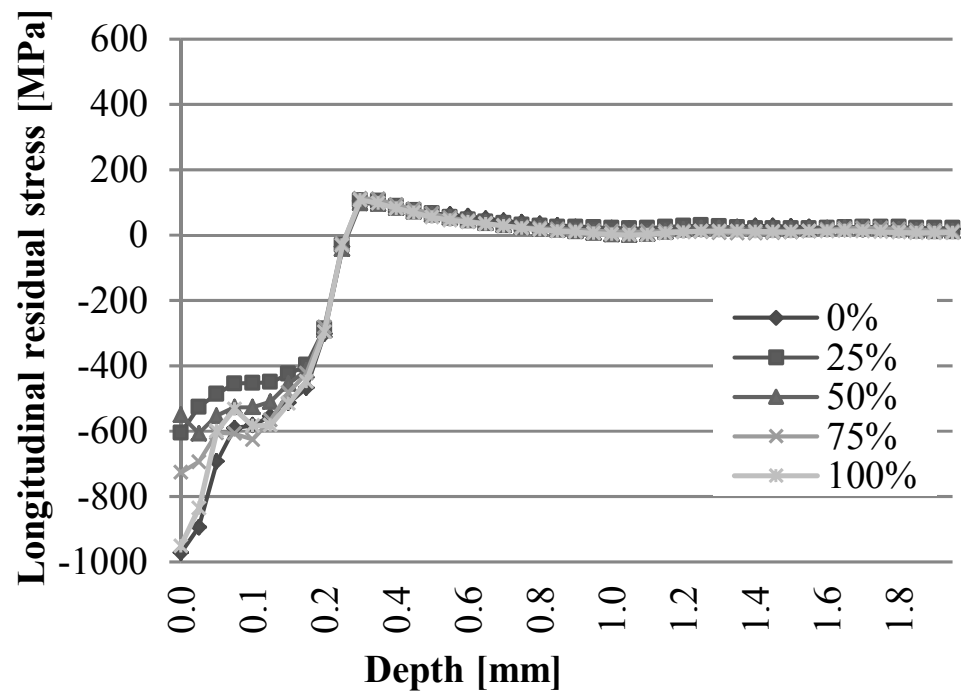

Fig. 11 Variation of the overlapping - longitudinal residual stresses.

Table 4 Variation of the number of overturns - area with compressive residual stresses.

\begin{tabular}{ll}
\hline Area with compressive residual stresses \\
\hline 0 overturn & $142 \mathrm{MPa} \cdot \mathrm{mm}$ \\
1 overturn & $179 \mathrm{MPa} \cdot \mathrm{mm}$ \\
2 overturns & $200 \mathrm{MPa} \cdot \mathrm{mm}$ \\
3 overturns & $224 \mathrm{MPa} \cdot \mathrm{mm}$ \\
5 overturns & $295 \mathrm{MPa} \cdot \mathrm{mm}$ \\
\hline
\end{tabular}

4.3. Effect of the Variation of the Overlapping on the Depth Distribution of Induced Residual Stresses

The last result shows the depth distribution of the longitudinal residual stresses in variation of the percentage of the overlapping. One non-expected observation is the depth distribution for $0 \%$ and for $100 \%$ overlapping. Though, higher maximum of the achieved residual stresses for $100 \%$ overlapping is expected, as the $100 \%$ overlapping means a repetition of the DR process on the same treatment area. Nevertheless, the residual stress depth profile for $100 \%$ overlapping remains relatively the same as those with $0 \%$ overlapping. An explanation of this result can be found in the applied on the DR sphere 
boundary conditions. In order to ensure a smooth treatment, after the application of the DR force, the DR sphere was fixed in vertical $(\mathrm{Y})$ direction. This prevented the deeper penetration of the sphere, when applying $100 \%$ overlapping.

\section{Discussion and Conclusions}

In this paper a FE Modelling of the mechanical surface treatment process Deep Rolling for AISI 4140 steel using the software ABAQUS 6.13 was shown. The explicit module of the program was employed to model the full 3D dynamic representation of the process. Several input parameters, like the applied normal force, the number of overturns and the overlapping percentage, were varied. Their influence on the induced longitudinal and transverse residual stresses was described.

The application of a higher DR force leads to an increasing of the compressive residual stress maximum. It is visible that a saturation level is reached when treating with 75 and $100 \mathrm{~N}$, where the application of a higher DR force does not lead to an increasing of the compressive residual stress maximum. Another tendency is also visible - with increasing the DR force the layer with compressive residual stresses also increase.

The observations of the overturns variation show that the magnitude of the induced compressive residual stresses remains the same and even decreases when increasing the number of overturns. Still, the layer with compressive residual stresses is thicker if a higher number of overturns is applied.

The overlapping variation shows that a certain overlapping percentage (from 25 to $75 \%$ ) leads to a relaxation of the induced maximal compressive residual stresses. Still, their depth is not influenced by the change of the overlapping percentage. The non-expected result for $0 \%$ and $100 \%$ overlapping is due to the fixation of the DR sphere in $\mathrm{Y}$ direction after application of the DR force, which prevents the deeper penetration of the DR sphere and respectively the inducement of higher compressive residual stresses. The fixation must be removed, as under realistic conditions the DR sphere is hydrostaticallyor spring supported and has a free play in $\mathrm{Y}$ direction.

Overall, the presented model shows results which are in good agreement with the existing literature. Several articles [6, 21] present measurements of the DR residual stress profiles which quantitatively fit to the presented in this paper. Still, the results can be only qualitatively evaluated as they differ from the used material and the process parameters. Therefore, a comparison with experimental data in order to confirm the presented results is highly appreciated. Some critical issues of the FE model are for example the material model. In the literature there are proofs that the bi-linear kinematic hardening model can properly represent the material behaviour, but the linear simplification can be a source of uncertainty. Another improvement issue is the mesh density - in the results a steep stress gradient near the treated surface is observed and there, the mesh density can be increased to assure more measurement points.

\section{References}

[1] Mader, S. and Klocke, F. 2005. "Fundamentals of the Deep Rolling of Compressor Blades for Turbo Aircraft." In Conference Proceedings: ICSP-9, 125-30.

[2] Thum, A. and Bautz, W. 1935. "Zur Frage der Formziffer." Forschung im Ingenieurwesen 6 (121): 269-73.

[3] Oberflächen-Feinwalzen 1983. VDI-Richtlinie 3177, Düsseldorf.

[4] Jung, U., Kaiser, B., Kloos, K. and Berger, C. 1996. "Festwalz-Eigenspannungen per Computer Simulation Bestimmen." Materialwissenschaft und Werkstofftechnik 27: $159-64$.

[5] Schulze, V. 2006. "Surface Layer States after Mechanical Surface Treatments" Schulze, V. (ed.): Modern Mechanical Surface Treatment - States, Stability, Effects. WILEY-VCH Verlag GmbH \& Co. KGaA, 95. Weinheim: 18.

[6] Wong, C., Hartawan, A. and Teo, W. 2014. "Deep Cold Rolling of Features on Aero-Engine Components." In 2nd CIRP Conference on Surface Integrity Procedia CIRP 13: 350-4.

[7] Manoucherhrifar, A. and Alasvand, K. 2009. "Finite 
Element Simulation of Deep Rolling and Evaluate the Influence of Parameters on Residual Stress." Recent Researches in Engineering Mechanics, Urban \& Naval Transportation and Tourism 61-7.

[8] Bärker, V., Klocke, F., Wegner, H., Timmer, A., Grzhibovskis, R. and Rjasanow, S. 2010. "Analysis of the Deep Rolling Process on Turbine Blades using the FEM/BEM-Coupling." IOP Conference Series: Materials Science and Engineering 10 (1): 1-10.

[9] Abrao, A. M., Denkena, B. and Breidenstein, B. 2014. "Surface and Subsurface Alterations INduced by Deep Rolling of Hardened AISI 1060 Steel." Production Engineering Research and Development 8: 551-8.

[10] Röttger, K., Wilcke, G. and Mader, S. 2005. "Deep Rolling - A Technology for Efficient Lightweight Design.” Materials Science and Engineering Technology 36 (6): 270-4.

[11] Mayer, H., Achmus, C. and Pyzalla, A. 2000. "Increase of Fatigue Life Strength of Crankshafts by Deep-Rolling and Induction Hardening Induced Beneficial Residual Stresses States - Experimental Determination and FEM Simulation." Deutsche Gesellschaft für Materialkunde e.V. Accessed Sep. 25, 2015. http://www.dgm.de/download/tg/523/523_0631.pdf.

[12] Altenberger, I. 2005. "Deep Rolling - The Past, the Present and the Future." In Proceedings of 9th International Conference on Shot Peening. 144-55.

[13] Werkzeug Bürger + Maluck GmbH. 2015. "Deep Rolling Applications, Application Examples.” Accessed Oct. 29, 2015. http://www.ecoroll.de/anwendungen/sonstiges/ schweissnaehte.html.

[14] ECOROLL AG. 2015. "Applications, Other, Weld Seams." Accessed Oct. 29, 2015. http://www.ecoroll.de/anwendungen/sonstiges/schweissn aehte.html.

[15] Huang, X. 2005. “A General Autofrettage Model of a Thick-Walled Cylinder Based on Tensile - Compressive Stress - Strain Curve of a Material." The Journal of Strain Analysis for Engineering Design 40 (6): 599-607.

[16] Bauschinger, J. 1886. "On the Change of the Elastic Limit and Strength of Iron and Steel by Stretching and Squeezing, by Heating and Cooling and Often Repeated Stress." Information from the Mechanical-technical laboratory. K. TH Munich 13.

[17] Demurger, J., Forestier, R., Kieber, B. and Lasne, P. 2006. "Deep Rolling Process Simulation: Impact of Kinematic Hardening on Residual Stresses." In Conference Proceedings of the 9th, International ESAFORM Conference on Material Forming 463-6.

[18] Brünnet, H., Yi, I. and Bähre, D. 2011. "Modeling of Residual Stresses and Shape Deviations along the Process Chain of Autofrettaged Components." Journal of Materials Science and Engineering A Volume 1/7A: 915-36.

[19] Brünnet, H. and Bähre, D. 2013. "Simulation of Beneficial Compressive Residual Stress Fields as Design Tool in Manufacturing Internally Pressurized Parts." In Proceedings of Simulia Community Conference 2013, Vienna, (Austria).

[20] ABAQUS Analysis User's Guide 6.14. "Using the Basic Coulomb friction model." Chapter 37.1.5.

[21] Beghini, M., Bertini, L., Monelli, B. D., Santus, C. and Bandini, M. 2014. "Experimental Parameter Sensitivity Analysis of Residual Stresses Induced by Deep Rolling on 7075-T6 Aluminium Alloy." Surface and Coatings Technology Volume 254: 175-86. 